\title{
Optimization of Process Variables in the Synthesis of Tributyl Citrate Using a Polyvinylpolypyrrolidone-Supported Brønsted Acidic Ionic Liquid Catalyst
}

\author{
Song Wang $\mathbb{D}^{D}$, Lanlan Xu, Linlin Xu, Chengcheng Tian, and Yinyan Guan \\ School of Science, Shenyang University of Technology, Shenyang, Liaoning 110870, China \\ Correspondence should be addressed to Song Wang; 1259669134@qq.com
}

Received 6 March 2018; Revised 24 May 2018; Accepted 14 June 2018; Published 15 July 2018

Academic Editor: Marta Fernández-García

Copyright (C) 2018 Song Wang et al. This is an open access article distributed under the Creative Commons Attribution License, which permits unrestricted use, distribution, and reproduction in any medium, provided the original work is properly cited.

\begin{abstract}
A polyvinylpolypyrrolidone- (PVPP-) supported Brønsted acidic ionic liquid catalyst $\left(\left[\mathrm{BsPVPP}^{\mathrm{P}} \mathrm{HSO}_{4}\right)\right.$ was synthesized by the reaction between $\mathrm{SO}_{3} \mathrm{H}$-functionalized PVPP and $\mathrm{H}_{2} \mathrm{SO}_{4}$. The prepared catalyst was characterized by IR, XRD, FESEM, TG, and DSC. The catalytic activity of [BsPVPP] $\mathrm{HSO}_{4}$ in the preparation of tributyl citrate (TBC) by the esterification reaction between citric acid and n-butanol was investigated. Response surface methodology (RSM) was applied to optimize the process variables of the esterification reaction. The variables, including the reaction time, the n-butanol-to-citric acid mole ratio, the reaction temperature, and the catalyst amount, were optimized by a Box-Behnken design. Under optimized conditions, with a n-butanolto-citric acid mole ratio of $5.2: 1$ and a reaction temperature of $120^{\circ} \mathrm{C}$, the TBC yield reached $92.9 \%$ within $5.5 \mathrm{~h}$ in the presence of $6.6 \mathrm{wt} \%$ of catalyst; this result is in good agreement with the values predicted by the mathematical model. Moreover, the catalyst could be recycled four times with high catalytic activity.
\end{abstract}

\section{Introduction}

Tributyl citrate (TBC) is usually applied as a thermally stable, high-performance, and nontoxic plasticizer in toys, medical products, biodegradable polymers, and food additives [1-4]. Moreover, TBC is a biorenewable and sustainable plasticizer. This is due to the fact that the raw materials applied in the preparation of TBC can be gotten from the fermentation processes using renewable resources $[5,6]$. Thus, the preparation of TBC has drawn much attention. Traditionally, the catalyst applied in the preparation of TBC is sulfuric acid, a homogeneous catalyst that has some disadvantages such as high by-product generation, equipment corrosion, and environmental pollutions. To solve the above problems, the heterogeneous acid catalysts that offer easy separation, high catalytic activity, and environmental friendliness were developed for TBC preparation. Metal oxide and zeolitesupported catalysts have attracted more and more attention [7-10]. Nevertheless, these heterogeneous catalysts have some disadvantages, including low product selectivity, high mass transfer resistance, and rapid deactivation during the esterification reaction.

Because of their excellent thermal stability, negligible volatility, and tunable acidity, acidic ionic liquids (ILs) have been utilized as environmental friendly reagents for organic reactions [11-17]. However, ILs have been proved to have some disadvantages, including poor reusability, high viscosity, and large amount requirement for the reaction [18-20]. Heterogeneous polymer-supported catalysts had been developed to solve these problems because of their environmentally friendly characteristics, such as excellent chemical stability, efficient recover ability, and recyclability [21-24]. These properties make polymer-supported ILs suitable candidates for catalytic reactions. Polyvinylpolypyrrolidone (PVPP), a commercialized cross-linked polymer adsorbent, has been used as a polymer support since it has many advantages, including being nontoxic, high adsorption capacity, and being insoluble in all types of solvents $[25,26]$. Moreover, it has been demonstrated that PVPP-supported catalysts have high catalytic ability and reusability in organic reactions [27-29]. 
Response surface methodology (RSM) is a frequently used mathematical method which can be applied to find the optimum reaction conditions by investigating the relationships between explanatory and response variables [30-32]. Therefore, in the present work, a polymer-supported Brønsted acidic IL catalyst, [BsPVPP] $\mathrm{HSO}_{4}$, was applied in the preparation of TBC by the esterification of $n$-butanol with citric acid for the first time. The effect of reaction conditions on the citric acid conversion and TBC yield was investigated thoroughly (single factor). Based on the results of the single-factor investigation, the reaction conditions were further optimized to increase the TBC yield by applying $\mathrm{RSM}$, and then the optimum reaction conditions were confirmed (multifactors). Additionally, the recyclability of $[\mathrm{BsPVPP}] \mathrm{HSO}_{4}$ was also discussed.

\section{Experimental Approach}

2.1. Materials. PVPP was purchased from Gobekie New Materials Science \& Technology Co. Ltd., Shanghai, China. 1,4-Butane sultone (BS) (99\%), toluene, sulfuric acid (98\%), ethyl acetate, and methanol (AR grade) were purchased from Sinopharm Chemical Reagent Co. and used without purification.

\subsection{Catalyst Preparation}

2.2.1. Preparation of $[\mathrm{Bs} P V P P] \mathrm{HSO}_{4}$. [BsPVPP $] \mathrm{HSO}_{4}$ was prepared as reported in previous publications with a little modification $[28,33]$. In a typical preparation procedure, PVPP powder ( $10 \mathrm{~g}$, monomer molar quantity was $0.09 \mathrm{~mol})$ was put into toluene under vigorous stirring, and after a while, BS $(6.8 \mathrm{~g}, 0.05 \mathrm{~mol})$ was introduced into the mixture. The mixture was heated to $80^{\circ} \mathrm{C}$ and stirred in a $100 \mathrm{~mL}$ flask for $24 \mathrm{~h}$ continuously. The resulting solid zwitterion was recovered by filtration and washed with ethyl acetate to completely remove the unreacted reactant and then dried at $60^{\circ} \mathrm{C}$ under vacuum overnight. Then, sulfuric acid $(5.0 \mathrm{~g}, 0.05 \mathrm{~mol})$ was added to the respective zwitterion dispersed in ethyl acetate at $0^{\circ} \mathrm{C}$ and stirred for $24 \mathrm{~h}$. After filtration, the residue was first washed three times with methanol and then dried under vacuum overnight.

2.2.2. Preparation of 1-Sulfobutyl-3-methylimidazolium $p$ Toluenesulfonate ([BsMIM]PTSA). Methylimidazole (9.1 g, $0.11 \mathrm{~mol})$ and BS $(13.6 \mathrm{~g}, 0.10 \mathrm{~mol})$ were added into a $100 \mathrm{~mL}$ round-bottom flask, where ethyl acetate was added later as a solvent. This flask was magnetically stirred at $40^{\circ} \mathrm{C}$ for $24 \mathrm{~h}$ until a solid zwitterion formed which was filtered, washed three times with diethyl ether to remove nonionic components, and then dried in vacuum. Then, p-toluenesulfonic acid (PTSA, $17.2 \mathrm{~g}, 0.1 \mathrm{~mol}$ ) was introduced to the respective zwitterion dissolved in water and stirred at $80^{\circ} \mathrm{C}$ for $8 \mathrm{~h}$. Finally, the product was obtained by vacuum drying.

2.3. Catalyst Characterization. The loading of the IL on the PVPP support was determined by elution experiment (supporting information). IR investigations were carried out on a Prestige-21 FTIR absorption spectrometer using $\mathrm{KBr}$ optics (Figure S1). Thermal decomposition of catalyst was investigated by a TA Q50 thermogravimetric instrument in $\mathrm{N}_{2}$ with a heating rate of $10^{\circ} \mathrm{C} / \mathrm{min}$ (Figure S2). Morphologies of [BsPVPP] $\mathrm{HSO}_{4}$ and PVPP were observed by a Hitachi SU8000 field emission scanning electron microscope equipped with energy-dispersive spectroscope (Figure S3). The element analysis of the catalyst was investigated via energy-dispersive spectroscope (Table S1), which has been proved to be a useful method to testify the chemical composition of the catalysts $[34,35]$. The XRD measurement was conducted by a Shimadzu XRD-6000 apparatus with a scanning speed of $5^{\circ} \cdot \mathrm{min}^{-1}$ (Figure S4). The differential scanning calorimetry (DSC) examination was performed using a TA Q20 instrument with a heating rate of $5^{\circ} \mathrm{C} / \mathrm{min}$ in the temperature range of $-80^{\circ} \mathrm{C}$ to $200^{\circ} \mathrm{C}$ as reported in literature [36] (Figure S5). NMR measurements were conducted on a Bruker AV 500 spectrometer (supporting information).

2.4. Esterification of Citric Acid with n-Butanol and Analysis. Preparation of TBC by the esterification reaction of citric acid with n-butanol was performed in a $100 \mathrm{~mL} 3$-necked flask equipped with a thermometer, a magnetic stirrer, and a segregator coupled with a reflux condenser at normal pressure (Scheme 1).

The typical reaction was applied as follows: citric acid (12.6 g, $0.06 \mathrm{~mol}), \mathrm{n}$-butanol $(22.2 \mathrm{~g}, 0.3 \mathrm{~mol})$, and [BsPVPP] $\mathrm{HSO}_{4}$ $(2.1 \mathrm{~g}, 6 \mathrm{wt} \%$ of raw material) were introduced into the flask. The reaction mixture was heated at $115^{\circ} \mathrm{C}$ with vigorous stirring for $5 \mathrm{~h}$. The conditions of the reaction system before and after the centrifugation separation were shown in Figure S6. After the reaction was completed, the product was investigated by gas chromatography (Agilent GC-7890A) using a Thermo TG-SQC capillary column $(15 \mathrm{~m} \times 0.25 \mathrm{~mm} \times 0.25 \mu \mathrm{m})$, which equipped with a flame ionization detector (FID). Acid value was determined by titration according to the procedures described in the literature [29]. Citric acid conversion and the TBC yield were calculated using the following equations [9]:

$$
\begin{aligned}
\mathrm{TBC} \text { yield } & =\frac{\text { actual amount of } \mathrm{TBC} \text { analyzed by GC }}{\text { theoretical amount of } \mathrm{TBC} \text { calculated from reactants }} \times 100 \%, \\
\text { citric acid conversion } & =\frac{\text { acid value before reaction }- \text { acid value after reaction }}{\text { acid value before reaction }} \times 100 \% .
\end{aligned}
$$




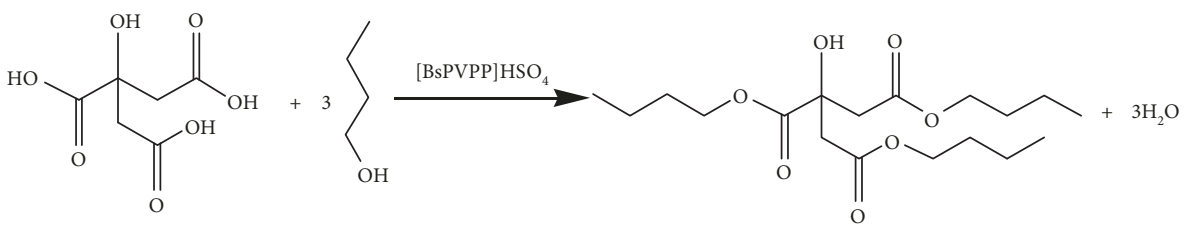

Scheme 1: Esterification of citric acid with n-butanol.

2.5. Response Surface Methodology. To optimize the reaction conditions for [BsPVPP $] \mathrm{HSO}_{4}$-catalyzed esterification reaction of n-butanol with citric acid, a three-level and four-factorial Box-Behnken design was designed to investigate the influences of the reaction conditions on the TBC yield. Esterification reactions using various molar ratios of n-butanol to citric acid (4.5-5.5), reaction temperatures $\left(110-126^{\circ} \mathrm{C}\right)$, catalyst amounts $(4 \%-8 \%)$, and reaction times (4-6h) were carefully investigated. Not only the variables and the levels of them but also the real and coded values are shown in Table 1. TBC yield was the response value of the designed experiment.

The relationship between the TBC yield and reaction factors was expressed using the following quadratic equation (2):

$$
\begin{aligned}
Y= & \beta_{0}+\beta_{1} X_{1}+\beta_{2} X_{2}+\beta_{3} X_{3}+\beta_{4} X_{4}+\beta_{11} X_{1}^{2}+\beta_{22} X_{2}^{2} \\
& +\beta_{33} X_{3}^{2}+\beta_{44} X_{4}^{2}+\beta_{12} X_{1} X_{2}+\beta_{13} X_{1} X_{3}+\beta_{14} X_{1} X_{4} \\
& +\beta_{23} X_{2} X_{3}+\beta_{24} X_{2} X_{4}+\beta_{34} X_{3} X_{4},
\end{aligned}
$$

where $Y$ is the TBC yield; $X_{1}$ to $X_{4}$ are corresponding parameters shown in Table 1; the meaning of other parameters are clearly shown in literature [30]. All of these parameters were obtained from the whole experimental results. The fitted equation was always expressed as response surface plots to demonstrate the relationship between the response and experimental levels of each variable. By this way, the optimal reaction conditions can be confirmed. Meanwhile, the coefficient of determination, $R_{2}$, was usually applied to determine the general ability and accuracy of the multiple regression models. Stat-Ease Design Expert (Version 8. 0. 6, Stat-Ease Inc., USA) was used to establish the mathematical model, generate the three-dimensional response surface, and determine the optimal reaction conditions.

\section{Results and Discussion}

3.1. Comparison of the Catalytic Activities of Different Catalysts. The catalytic performance of different catalysts for the esterification reaction of n-butanol with citric acid is presented in Table 2.

Based on the proton acid catalytic mechanism (Scheme S1), supported IL catalysts can catalyze the esterification reactions as efficient as inorganic acid and acidic IL [8, 37, 38]. The catalytic ability of inorganic acid, acidic IL, and $[\mathrm{BsPVPP}] \mathrm{HSO}_{4}$ was compared. It can be observed from Table 2 that, without using catalysts, the TBC yield only reached $12.5 \%$ (entry 1 ). When $\mathrm{H}_{2} \mathrm{SO}_{4}$ and PTSA were used
TABLE 1: Coded values of the variables used in the experimental design.

\begin{tabular}{lcccc}
\hline \multirow{2}{*}{ Variables } & \multirow{2}{*}{ Symbols } & \multicolumn{3}{c}{ Variable levels } \\
& & -1 & 0 & 1 \\
\hline Mole ratio of n-butanol to citric acid & $X_{1}$ & 4.5 & 5.0 & 5.5 \\
Reaction temperature $\left({ }^{\circ} \mathrm{C}\right)$ & $X_{2}$ & 110 & 118 & 126 \\
Amount of catalyst $(\mathrm{wt} \%)$ & $X_{3}$ & 4 & 6 & 8 \\
Reaction time $(\mathrm{h})$ & $X_{4}$ & 4 & 5 & 6 \\
\hline
\end{tabular}

\begin{tabular}{|c|c|c|c|}
\hline Entry & Catalyst $^{\mathrm{a}}$ & Conversion $(\%)^{\mathrm{b}}$ & Yield $(\%)^{\mathrm{c}}$ \\
\hline 1 & - & 59.7 & 12.5 \\
\hline 2 & $\mathrm{H}_{2} \mathrm{SO}_{4}$ & 96.4 & 91.3 \\
\hline 3 & PTSA & 96.5 & 92.2 \\
\hline 4 & [BsMIM]PTSA & 96.1 & 91.9 \\
\hline 5 & {$[\mathrm{BsPVPP}] \mathrm{HSO}_{4}$} & 96.9 & 92.1 \\
\hline
\end{tabular}

TABLE 2: Esterification of citric acid with n-butanol using different catalysts.

a Reaction conditions: n-butanol $(0.3 \mathrm{~mol})$, citric acid $(0.06 \mathrm{~mol})$, [BsPVPP] $\mathrm{HSO}_{4}(6 \mathrm{wt} \%)$, and $115^{\circ} \mathrm{C}$ for $5 \mathrm{~h} . \mathrm{H}_{2} \mathrm{SO}_{4}, \mathrm{H}_{3} \mathrm{PW}_{12} \mathrm{O}_{40}$, and [BsMIM]PTSA were used in a similar proton content as $[\mathrm{BsPVPP}] \mathrm{HSO}_{4}$. ${ }^{\mathrm{b}}$ Citric acid conversion. ${ }^{\mathrm{C}} \mathrm{TBC}$ yield.

as catalysts in esterification, homogeneous reaction systems were formed and all exhibited a high citric acid conversion, above 96\%, and TBC yield, above 91\% (entries 2 and 3). However, the homogeneous reaction systems made the separation of $\mathrm{H}_{2} \mathrm{SO}_{4}$ and PTSA from the product difficult. When [BsMIM]PTSA was used as a catalyst, a liquid-liquid heterogeneous system was formed after the reaction. The citric acid conversion was $96.1 \%$, and the TBC yield was $91.9 \%$ (entry 4). However, [BsMIM]PTSA was easily attached to the bottom of the flask like a gelatinous liquid, which can greatly reduce its reusability. [BsPVPP] $\mathrm{HSO}_{4}$ is a heterogeneous catalyst, when it was used, a liquid-solid reaction system formed and a high citric acid conversion, $96.9 \%$, and good TBC yield, 92.1\%, were obtained (entry 5). Moreover, [BsPVPP $] \mathrm{HSO}_{4}$ could be easily separated by vacuum filtration and then reused. Thus, [BsPVPP] $\mathrm{HSO}_{4}$ was used as the catalyst to optimize various reaction parameters in the esterification of n-butanol with citric acid.

3.2. Effect of the Molar Ratio of n-Butanol to Citric Acid. Excessive addition of the $n$-butanol reactant was important for the esterification reaction because it could increase the 


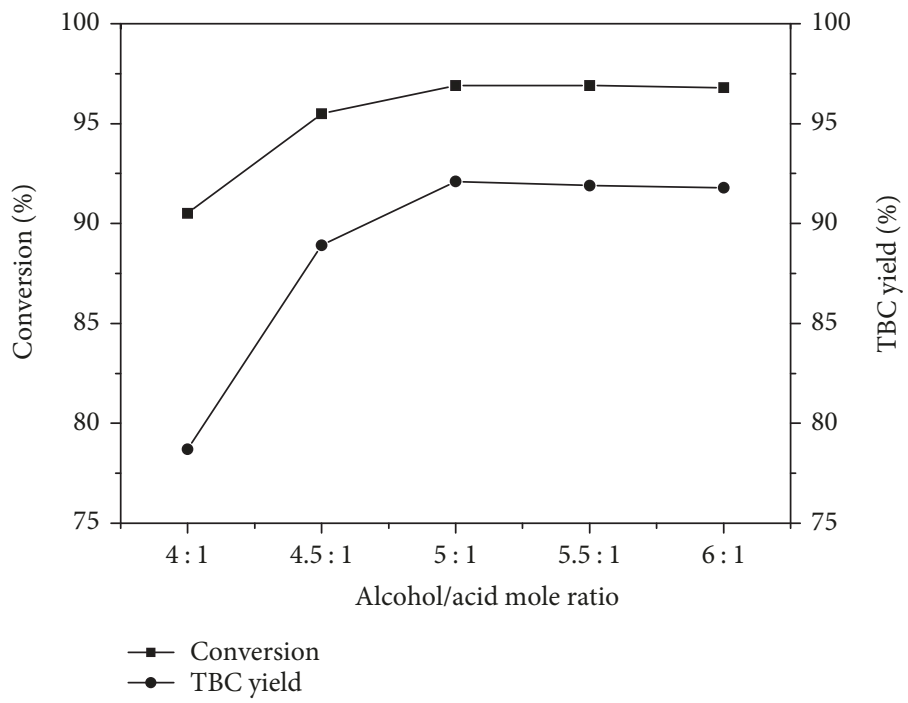

FIGURE 1: Effect of the n-butanol to citric acid molar ratio on the esterification of citric acid with n-butanol. Reaction conditions: citric acid, $0.06 \mathrm{~mol}$; n-butanol, $0.3 \mathrm{~mol}$; catalyst, $6 \mathrm{wt} \%$; temperature, $115^{\circ} \mathrm{C}$; time, $5 \mathrm{~h}$.

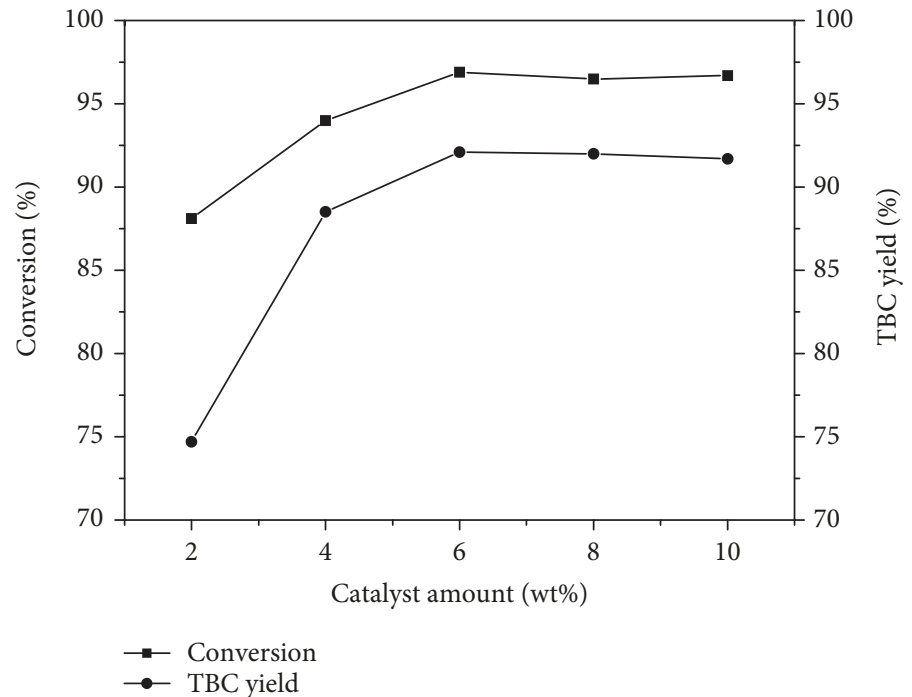

FIGURE 2: Effect of the catalyst amount on the esterification of citric acid with n-butanol. Reaction conditions: citric acid, 0.06 mol; $\mathrm{n}$-butanol, $0.3 \mathrm{~mol}$; temperature, $115^{\circ} \mathrm{C}$; time, $5 \mathrm{~h}$.

TBC yield and the citric acid conversion. Figure 1 shows the effect of the mole ratio of n-butanol to citric acid on the TBC yield and the citric acid conversion. It can be observed that the TBC yield and the citric acid conversion demonstrated a marked improvement with the increase of the molar ratio of n-butanol to citric acid from $4: 1$ to $5: 1$, and the maximum conversion of $96.9 \%$ and yield of $92.1 \%$ were achieved with a 5:1 molar ratio. This means that the excess nbutanol led to the shifting of the equilibrium towards the product side. Whereas, a further increase of the n-butanolto-citric acid mole ratio did not lead to a remarkable increase in the yield or conversion, most likely because the excessive n-butanol can dilute the concentration of citric acid and [BsPVPP] $\mathrm{HSO}_{4}$ too much. This phenomenon was in good agreement with other reported literature [9]. Taking the
TBC yield and the cost of n-butanol into account, the optimal molar ratio of $n$-butanol to citric acid was chosen to be $5: 1$.

3.3. Effect of the Amount of [BsPVPP]HSO ${ }_{4}$. The effect of the catalyst amount of [BsPVPP] $\mathrm{HSO}_{4}$ on its catalytic activity is illustrated in Figure 2. It can be observed that both the TBC yield and the citric acid conversion changed with increase in the amount of [BsPVPP] $\mathrm{HSO}_{4}$; a citric acid conversion rate of $96.9 \%$ and a TBC yield of $92.1 \%$ were obtained in the presence of $6 \%$ of $[\mathrm{BsPVPP}] \mathrm{HSO}_{4}$ within $5 \mathrm{~h}$ of the reaction. However, citric acid conversion and the TBC yield changed slightly when the catalyst amount was further increased. This phenomenon might be caused by the limitation of the chemical reaction equilibrium, as reported in literature [31]. Considering citric acid conversion, the TBC yield, 


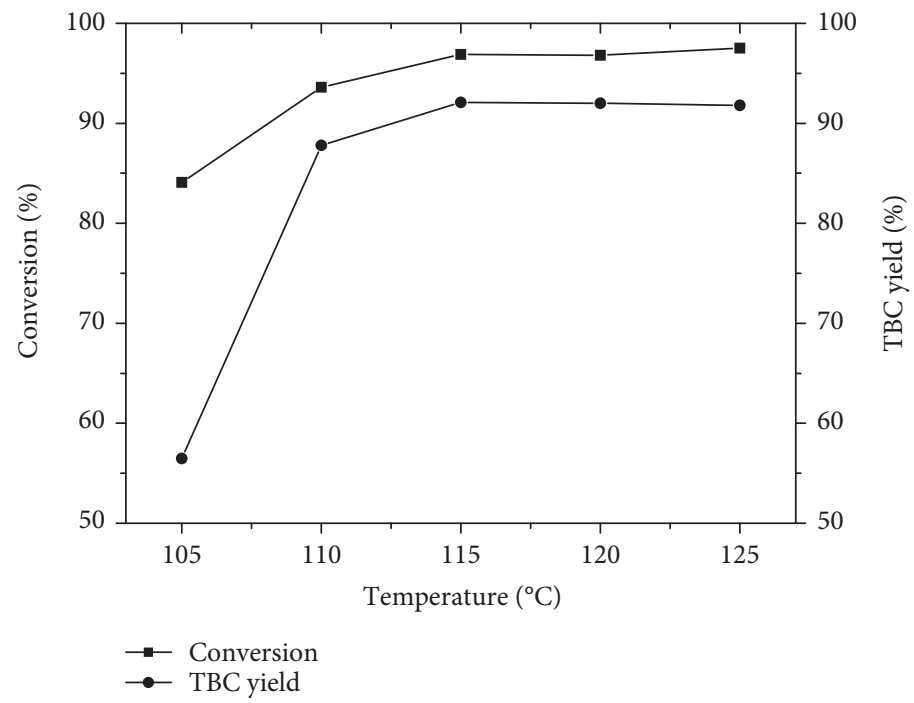

FIGURE 3: Effect of the reaction temperature on the esterification of citric acid with n-butanol. Reaction conditions: citric acid, 0.06 mol; n-butanol, $0.3 \mathrm{~mol}$; catalyst, $6 \mathrm{wt} \%$; time, $5 \mathrm{~h}$.

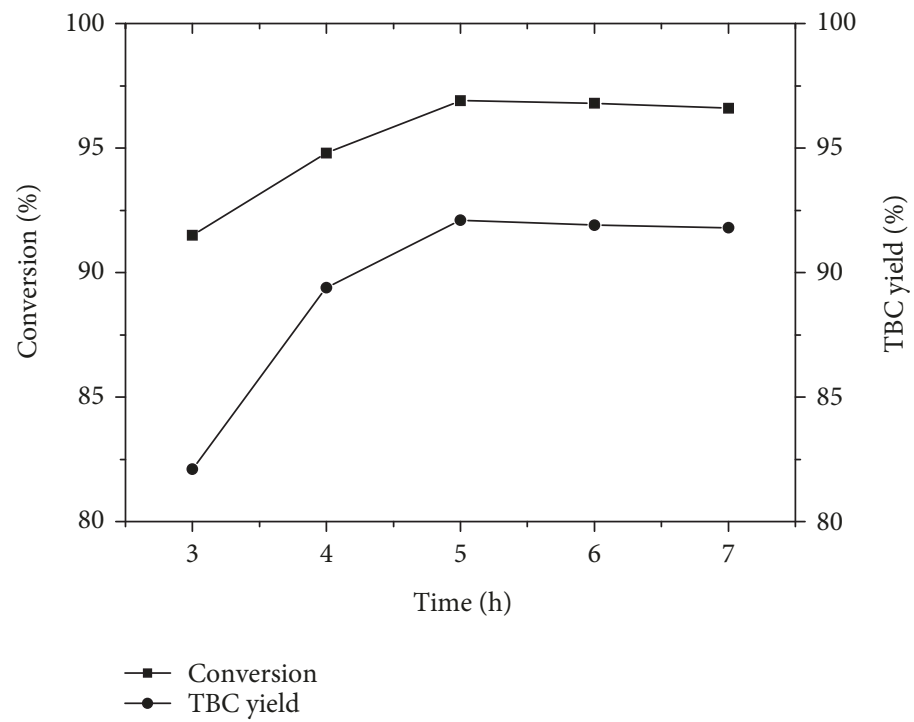

FIGURE 4: Effect of the reaction temperature on the esterification of citric acid with $\mathrm{n}$-butanol. Reaction conditions: citric acid, 0.06 mol; $\mathrm{n}$ butanol, $0.3 \mathrm{~mol}$; catalyst, $6 \mathrm{wt} \%$; temperature, $115^{\circ} \mathrm{C}$.

and reduced cost effectiveness, the optimum catalyst amount of [BsPVPP] $\mathrm{HSO}_{4}$ that was used in this reaction was $6 \%$.

3.4. Effect of the Reaction Temperature. Since high reaction temperature always accelerates the reaction rate and enhances the conversion of reagents, temperature is an important parameter for the esterification reaction and the effect of temperature must be investigated. Figure 3 depicts the changes of the TBC yield and the citric acid conversion at different temperatures. The results indicate that both the citric acid conversion and the TBC yield increased when the temperature increased from $105^{\circ} \mathrm{C}$ to $115^{\circ} \mathrm{C}$. The relatively high citric acid conversion of $96.9 \%$ and TBC yield of $92.1 \%$ were achieved at $115^{\circ} \mathrm{C}$. However, when the reaction temperature was higher than $115^{\circ} \mathrm{C}$, the $\mathrm{TBC}$ yield did not increase obviously. Thus, the optimum reaction temperature for the esterification reaction of n-butanol with citric acid was $115^{\circ} \mathrm{C}$.

3.5. Effect of the Reaction Time. The effect of the reaction time on the TBC yield and the citric acid conversion is presented in Figure 4. It can be observed that the TBC yield increased with the reaction time, that is, when the reaction time increased from $3 \mathrm{~h}$ to $5 \mathrm{~h}$, the TBC yield increased from less than $85 \%$ to $92.1 \%$. However, when the reaction time was more than $5 \mathrm{~h}$, the TBC yield decreased slightly. Therefore, the optimum reaction time was $5 \mathrm{~h}$ for this experiment.

3.6. Optimization of the [BsPVPP] $\mathrm{HSO}_{4}$-Catalyzed Esterification Process Using RSM. Based on single-factor experiments, RSM was applied to optimize the esterification reaction 
TABle 3: Experimental design and the results of the Box-Behnken design for the optimization process.

\begin{tabular}{|c|c|c|c|c|c|}
\hline \multirow{2}{*}{ Number } & \multicolumn{4}{|c|}{ Variable and level } & \multirow{2}{*}{ TBC yield (\%) } \\
\hline & $X_{1}$ & $X_{2}$ & $X_{3}$ & $X_{4}$ & \\
\hline 1 & -1 & 1 & 0 & 0 & 89.81 \\
\hline 2 & -1 & -1 & 0 & 0 & 85.23 \\
\hline 3 & -1 & 0 & 0 & -1 & 88.27 \\
\hline 4 & -1 & 0 & 1 & 0 & 88.57 \\
\hline 5 & -1 & 0 & -1 & 0 & 86.52 \\
\hline 6 & -1 & 0 & 0 & 1 & 88.90 \\
\hline 7 & 0 & 0 & 1 & -1 & 89.10 \\
\hline 8 & 0 & 0 & 1 & 1 & 91.93 \\
\hline 9 & 0 & 1 & 0 & -1 & 90.91 \\
\hline 10 & 0 & 0 & -1 & -1 & 87.72 \\
\hline 11 & 0 & 1 & -1 & 0 & 90.05 \\
\hline 12 & 0 & 1 & 1 & 0 & 91.79 \\
\hline 13 & 0 & 1 & 0 & 1 & 91.44 \\
\hline 14 & 0 & -1 & 0 & -1 & 87.15 \\
\hline 15 & 0 & 0 & -1 & 1 & 88.45 \\
\hline 16 & 0 & -1 & 1 & 0 & 88.20 \\
\hline 17 & 0 & -1 & 0 & 1 & 88.30 \\
\hline 18 & 0 & -1 & -1 & 0 & 85.80 \\
\hline 19 & 1 & -1 & 0 & 0 & 89.92 \\
\hline 20 & 1 & 0 & -1 & 0 & 90.12 \\
\hline 21 & 1 & 0 & 0 & -1 & 90.22 \\
\hline 22 & 1 & 1 & 0 & 0 & 91.23 \\
\hline 23 & 1 & 0 & 1 & 0 & 91.42 \\
\hline 24 & 1 & 0 & 0 & 1 & 91.61 \\
\hline 25 & 0 & 0 & 0 & 0 & 92.05 \\
\hline 26 & 0 & 0 & 0 & 0 & 92.43 \\
\hline 27 & 0 & 0 & 0 & 0 & 92.70 \\
\hline 28 & 0 & 0 & 0 & 0 & 92.24 \\
\hline 29 & 0 & 0 & 0 & 0 & 92.60 \\
\hline
\end{tabular}

conditions, fully considering multifactor interactions. Using the independent variables and the corresponding levels for the Box-Behnken design shown in Table 1, the experimental results are presented in Table 3 .

The following regression equation was generated from the application of RSM. It is an empirical relationship between the response variable, TBC yield, and the tested variables:

$$
\begin{gathered}
Y=92.40+1.44 X_{1}+1.72 X_{2}+1.03 X_{3}+0.61 X_{4} \\
-0.82 X_{1} X_{2}-0.19 X_{1} X_{3}+0.19 X_{1} X_{4}-0.16 X_{2} X_{3} \\
-0.15 X_{2} X_{4}+0.53 X_{3} X_{4}-1.50 X_{1}^{2}-1.75 X_{2}^{2} \\
-1.77 X_{3}^{2}-1.23 X_{4}^{2}, \\
R^{2}=0.98, \\
R_{\mathrm{adj}}{ }^{2}=0.97 .
\end{gathered}
$$

The regression result also shows that the value of the regression parameter, $R^{2}$, is close to 1 . This result indicates
TABle 4: Significance of the coefficient and the corresponding statistical analysis.

\begin{tabular}{lccccc}
\hline Source & Sum of squares & $\mathrm{df}$ & Mean square & $F$ value & $P$ value \\
\hline Model & 123.54 & 14 & 8.82 & 57.92 & $<0.0001$ \\
$X_{1}$ & 14.94 & 1 & 14.94 & 98.03 & $<0.0001$ \\
$X_{2}$ & 23.52 & 1 & 23.52 & 154.37 & $<0.0001$ \\
$X_{3}$ & 1.95 & 1 & 1.95 & 12.79 & 0.0030 \\
$X_{4}$ & 1.41 & 1 & 1.41 & 9.27 & 0.0087 \\
$X_{1} X_{2}$ & 2.67 & 1 & 2.67 & 17.55 & 0.0009 \\
$X_{1} X_{3}$ & 0.14 & 1 & 0.14 & 0.92 & 0.3530 \\
$X_{1} X_{4}$ & 0.14 & 1 & 0.14 & 0.95 & 0.3468 \\
$X_{2} X_{3}$ & 0.11 & 1 & 0.11 & 0.71 & 0.4121 \\
$X_{2} X_{4}$ & 0.096 & 1 & 0.096 & 0.63 & 0.4403 \\
$X_{3} X_{4}$ & 1.10 & 1 & 1.10 & 7.24 & 0.0176 \\
$X_{1}{ }^{2}$ & 14.63 & 1 & 14.63 & 96.05 & $<0.0001$ \\
$X_{2}{ }^{2}$ & 19.88 & 1 & 19.88 & 130.50 & $<0.0001$ \\
$X_{3}{ }^{2}$ & 20.34 & 1 & 20.34 & 133.50 & $<0.0001$ \\
$X_{4}{ }^{2}$ & 9.81 & 1 & 9.81 & 64.36 & $<0.0001$ \\
Residual & 2.13 & 14 & 0.15 & & \\
Lack of fit & 1.85 & 10 & 0.19 & 2.66 & 0.1793 \\
Pure error & 0.28 & 4 & 0.070 & & \\
Cor total & 125.67 & 28 & & & \\
\hline & & & & &
\end{tabular}

that there is a good fitness between the predicted values and experimental data. Thus, using this model to predict the TBC yield is reliable.

The significant test for each regression parameter involved in (3) and the variable analysis are presented in Table 4. The detailed three-dimensional response diagrams obtained from the predicted models are shown in Figure 5. As seen from Table 4 , the $P$ value $(<0.0001)$ of the model is very low, which indicates that the regression is statistically significant. Additionally, the $P$ values of $X_{1}(P<0.0001), X_{2}$ $(P<0.0001), X_{3}(P=0.0030)$, and $X_{4}(P=0.0087)$ were all lower than 0.05 , indicating the high significance of (3). This result also showed that the n-butanol-to-citric acid molar ratio and reaction temperature have a highly significant effect on the TBC yield. Moreover, the interactions between the factors of $X_{1} X_{2}(P=0.0009)$ and $X_{3} X_{4}(P=0.0176)$ were significant, that is, the interactions, including the molar ratio of $n$-butanol to citric acid-reaction temperature and amount of catalyst-reaction time, had a noticeable influence on the TBC yield.

Optimum reaction conditions can also be deduced from (3) by means of analyzing the multiparameter regression. Optimum parameters for the esterification reaction of $n$ butanol with citric acid were as follows: a n-butanol to citric acid mole ratio of $5.19: 1$, reaction temperature of $121.00^{\circ} \mathrm{C}$, catalyst amount of $6.60 \mathrm{wt} \%$, and reaction time of $5.32 \mathrm{~h}$. A maximum TBC yield of $93.2 \%$ was obtained with this set of optimum parameters. Considering the predicted value and actual experimental conditions, a n-butanol to citric acid 


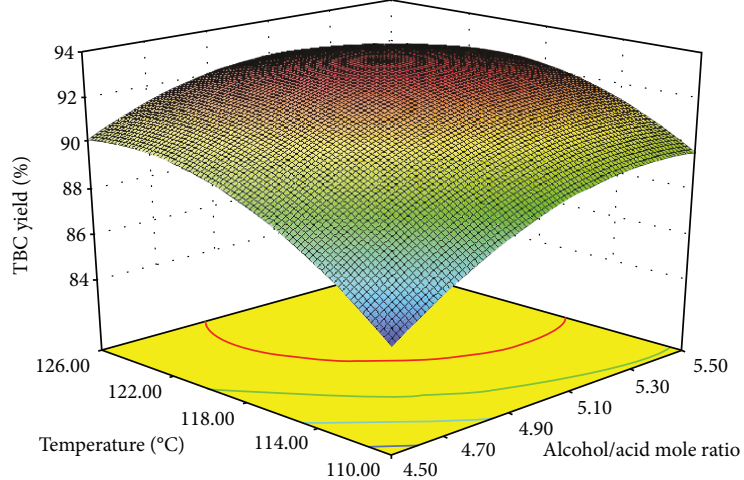

(a)

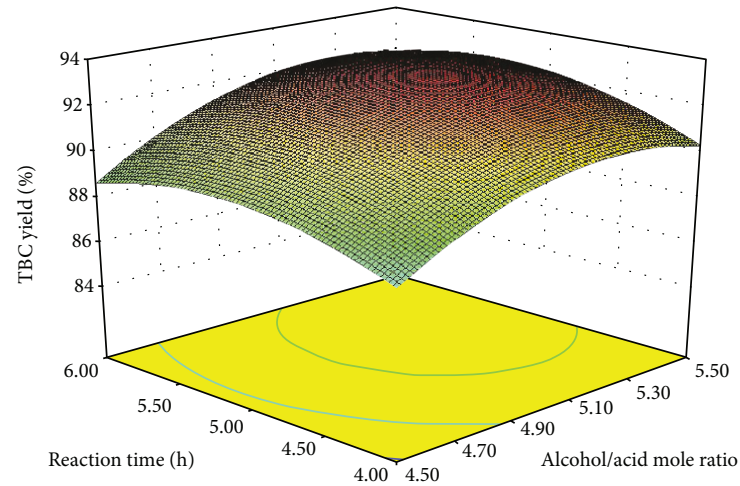

(c)

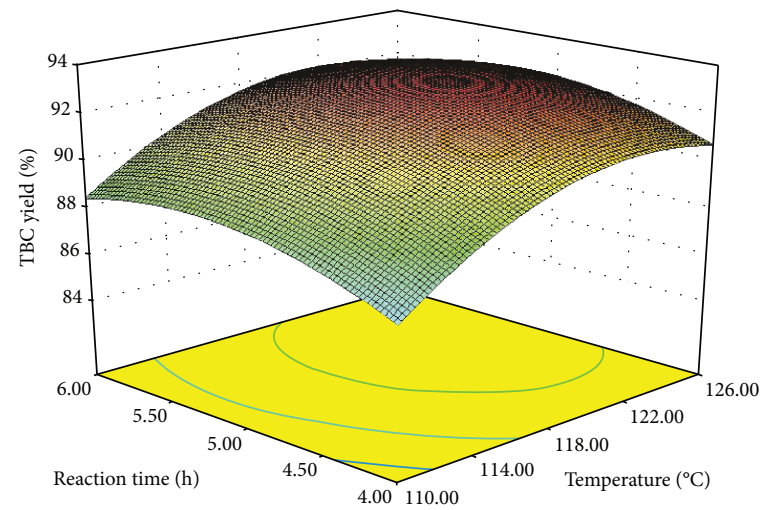

(e)

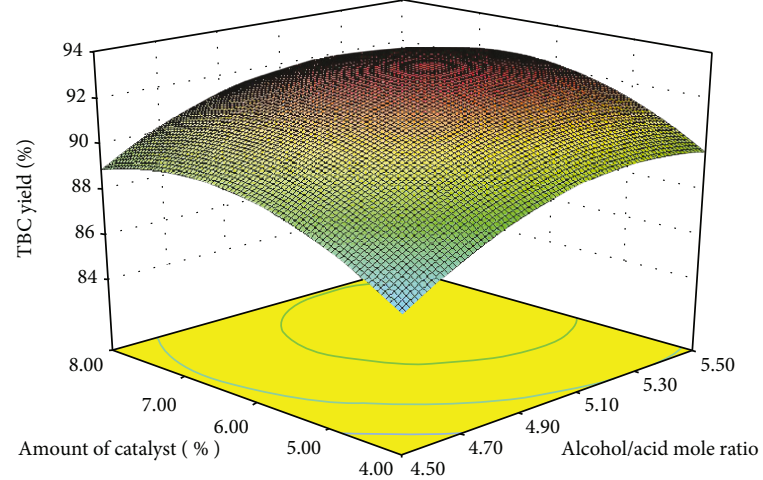

(b)

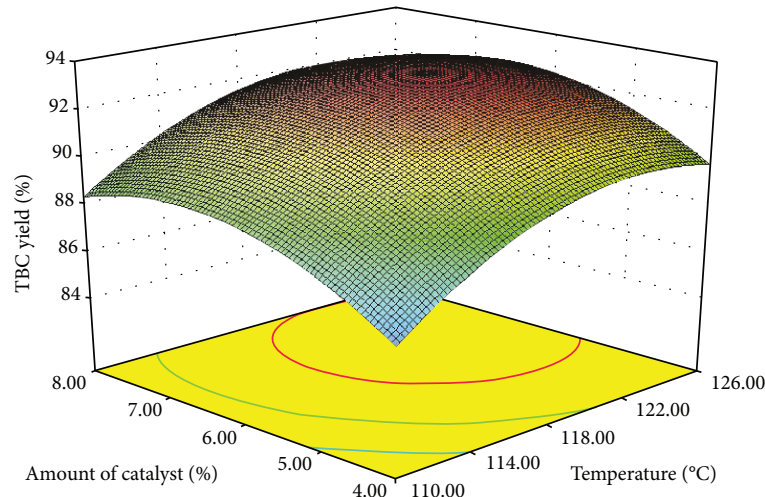

(d)

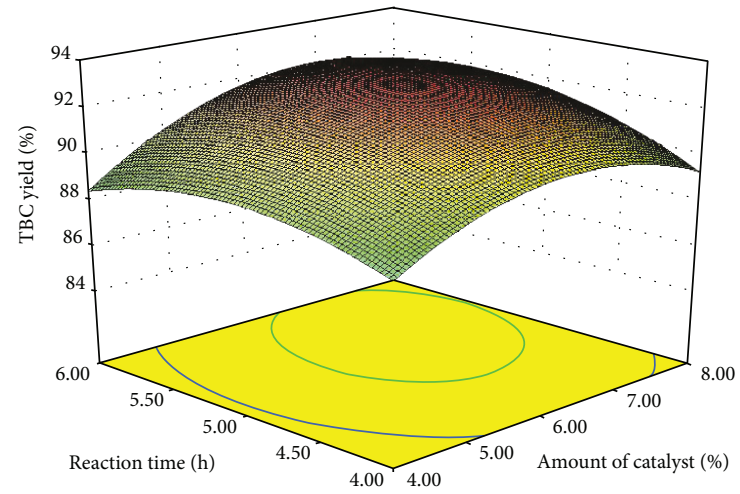

(f)

FIGURE 5: Response surface plots showing the predicted values of the TBC yield: effect of the (a) n-butanol-to-citric acid molar ratio and temperature, (b) n-butanol-to-citric acid molar ratio and amount of catalyst, (c) n-butanol-to-citric acid molar ratio and reaction time, (d) temperature and amount of catalyst, (e) temperature and reaction time, and (f) amount of catalyst and reaction time on the TBC yield. Other variables were kept at their central level.

mole ratio of $5.2: 1$, reaction temperature of $120^{\circ} \mathrm{C}$, catalyst amount of $6.6 \mathrm{wt} \%$, and reaction time of $5.5 \mathrm{~h}$ were selected. Three parallel experiments were performed under the above conditions to confirm the consistency of the predicted and measured data. The measured TBC yield was $92.9 \%$, which was almost the same with the predicted value, indicating agreement with the predicted model. Thus, the developed RSM model was adequately confirmed to depict the relationship between the variables and response in the synthesis of TBC.
3.7. Catalyst Reusability. The recyclability of [BsPVPP] $\mathrm{HSO}_{4}$ used in the reaction of n-butanol with citric acid was also investigated in this work. After each cycling reaction, $[\mathrm{BsPVPP}] \mathrm{HSO}_{4}$ was washed with diethyl ether and vacuum heated for $2 \mathrm{~h}$ at $80^{\circ} \mathrm{C}$. As seen in Figure 6, the citric acid conversion changed from $97.5 \%$ to $95.3 \%$ and the TBC yield changed from $92.9 \%$ to $90.1 \%$ after four cycles of recycling. Thus, the good recyclability reveals that $[\mathrm{BsPVPP}] \mathrm{HSO}_{4}$ is very stable for the esterification reaction of $n$-butanol with citric acid. 


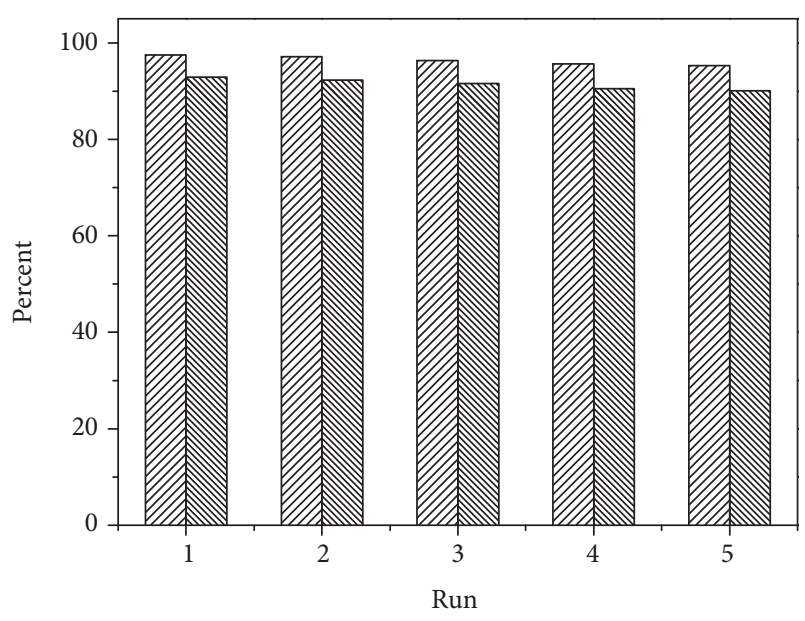

WIIIS Citric acid conversion

FIGURE 6: Reusability of the [BsPVPP $] \mathrm{HSO}_{4}$ catalyst for the esterification of citric acid with n-butanol. Reaction conditions: citric acid, $0.06 \mathrm{~mol}$; n-butanol, $0.31 \mathrm{~mol}$; catalyst, $6.6 \mathrm{wt} \%$; temperature, $120^{\circ} \mathrm{C}$; time, $5.5 \mathrm{~h}$.

\section{Conclusion}

A polymer-supported Brønsted acidic IL catalyst, [BsPVPP] $\mathrm{HSO}_{4}$, was synthesized and used for the esterification of n-butanol with citric acid. To optimize and study the effect of the process parameters, including the reactant mole ratio, reaction temperature, catalyst amount, and reaction time, on the TBC yield and the citric acid conversion, RSM was used based on single-factor experiments. According to the optimization results obtained with RSM, the optimum esterification conditions of n-butanol with citric acid are the n-butanol-to-citric acid mole ratio of $5.2: 1$, reaction time of $5.5 \mathrm{~h}$, reaction temperature of $120^{\circ} \mathrm{C}$, and catalyst amount of $6.6 \mathrm{wt} \%$. Under the optimized conditions, citric acid conversion and the TBC yield reached 97.5\% and $92.9 \%$, respectively. Moreover, [BsPVPP] $\mathrm{HSO}_{4}$ exhibited excellent reusability. After four cycles of recycling, both citric acid conversion and the TBC yield decreased by no more than $3 \%$. Thus, $[\mathrm{BsPVPP}] \mathrm{HSO}_{4}$ is an attractive candidate for industrial TBC synthesis.

\section{Data Availability}

The data used to support the findings of this study are available from the corresponding author upon request.

\section{Conflicts of Interest}

The authors declare that there is no conflict of interest regarding the publication of this paper.

\section{Acknowledgments}

This work was financially supported by the Shenyang Science and Technology Plan Projects (no. F15-199-1-12), the Key
Laboratory for Catalyst Synthesis Technology of Polymer of Liaoning Province, China (no. 2010-36), the Engineering Laboratory for Advanced Polymer Material of Liaoning Province, China (no. 2012-1139-15), and the Doctoral Scientific Research Foundation of Liaoning Province (no. 201601170). Authors would like to thank Dr. Zhihong Zhang and Dr. Patrick U. Okoye for their contribution to the paper.

\section{Supplementary Materials}

The supplementary materials include four aspects. Firstly, the procedure of the elution experiment was carefully described. Secondly, the FTIR, TGA, SEM, EDS, XRD, DSC, and NMR results about the catalyst were presented. Thirdly, the conditions of the reaction system before and after the centrifugation separation were shown. Lastly, the catalytic mechanism for esterification of citric acid with n-butanol over [BsPVPP] $\mathrm{HSO}_{4}$ catalyst was demonstrated. (Supplementary Materials)

\section{References}

[1] L. M. Rasmussen, N. Sen, X. Liu, and Z. R. Craig, "Effects of oral exposure to the phthalate substitute acetyl tributyl citrate on female reproduction in mice," Journal of Applied Toxicology, vol. 37, no. 6, pp. 668-675, 2017.

[2] Z. Liu, Y. Lei, Z. Hu, W. Kong, C. Zhou, and J. Lei, "Preparation, characterization and properties of poly(lactic acid)/ poly(1,4-butylene adipate) blends for biodegradable packaging materials," Macromolecular Research, vol. 25, no. 5, pp. 439445, 2017.

[3] M. Râpă, A. C. Miteluț, E. E. Tănase et al., "Influence of chitosan on mechanical, thermal, barrier and antimicrobial properties of PLA-biocomposites for food packaging," Composites Part B Engineering, vol. 102, pp. 112-121, 2016.

[4] T. T. Bui, G. Giovanoulis, A. P. Cousins, J. Magnér, I. T. Cousins, and C. A. de Wit, "Human exposure, hazard and risk of alternative plasticizers to phthalate esters," Science of the Total Environment, vol. 541, pp. 451-467, 2016.

[5] N. Abdehagh, F. H. Tezel, and J. Thibault, "Separation techniques in butanol production: challenges and developments," Biomass and Bioenergy, vol. 60, pp. 222-246, 2014.

[6] A. R. Angumeenal and D. Venkappayya, "An overview of citric acid production," LWT - Food Science and Technology, vol. 50, no. 2, pp. 367-370, 2013.

[7] K. Y. A. Lin, T. Y. Lin, Y. C. Lu, J. T. Lin, and Y. F. Lin, "Electrospun nanofiber of cobalt titanate perovskite as an enhanced heterogeneous catalyst for activating peroxymonosulfate in water," Chemical Engineering Science, vol. 168, pp. 372-379, 2017.

[8] K. Y. Nandiwale, P. Gogoi, and V. V. Bokade, "Catalytic upgrading of citric acid to environmental friendly tri-butyl citrate plasticizer over ultra stable phosphonated Y zeolite," Chemical Engineering Research and Design, vol. 98, pp. 212219, 2015.

[9] H. Yang, H. Song, H. Zhang, P. Chen, and Z. Zhao, "Esterification of citric acid with n-butanol over zirconium sulfate supported on molecular sieves," Journal of Molecular Catalysis A: Chemical, vol. 381, pp. 54-60, 2014. 
[10] S. X. Li, Y. R. Xu, and S. Wang, "Synthesis of TMPOT with $\mathrm{SO}_{4}{ }^{2-} / \mathrm{TiO}_{2}-\mathrm{HZSM}-5$ solid superacid catalyst," Journal of Shenyang University of Technology, vol. 37, pp. 231-235, 2015.

[11] A. A. Chaugule, A. H. Tamboli, and H. Kim, "Ionic liquid as a catalyst for utilization of carbon dioxide to production of linear and cyclic carbonate," Fuel, vol. 200, pp. 316-332, 2017.

[12] Z. P. Zhao, X. L. Wang, G. Y. Zhou, Y. Cao, P. Lu, and W. F. Liu, "Hydrolysis kinetics of inulin by imidazole-based acidic ionic liquid in aqueous media and bioethanol fermentation," Chemical Engineering Science, vol. 151, pp. 16-24, 2016.

[13] M. S. Fedoseev, M. S. Gruzdev, and L. F. Derzhavinskaya, “1Butyl-3-methylimidazolium salts as new catalysts to produce epoxy-anhydride polymers with improved properties," International Journal of Polymer Science, vol. 2014, Article ID 607341, 8 pages, 2014.

[14] C. Croitoru and S. Patachia, "Long-chain alkylimidazolium ionic liquid functionalization of cellulose nanofibers and their embedding in HDPE matrix," International Journal of Polymer Science, vol. 2016, Article ID 7432528, 9 pages, 2016.

[15] M. Yoshizawa and H. Ohno, "Anhydrous proton transport system based on zwitterionic liquid and HTFSI," Chemical Communications, vol. 16, no. 16, pp. 1828-1829, 2004.

[16] M. Yoshizawa-Fujita, N. Byrne, M. Forsyth, D. R. MacFarlane, and H. Ohno, "Proton transport properties in zwitterion blends with Brønsted acids," Journal of Physical Chemistry B, vol. 114, no. 49, pp. 16373-16380, 2010.

[17] A. C. Cole, J. L. Jensen, I. Ntai et al., "Novel Brønsted acidic ionic liquids and their use as dual solvent-catalysts," Journal of the American Chemical Society, vol. 124, no. 21, pp. 59625963, 2002.

[18] P. Lu, Z. P. Zhao, X. Y. Wang, G. J. Lan, and X. L. Wang, "Understanding effect of molecular structure of imidazolebased ionic liquids on catalytic performance for biomass inulin hydrolysis," Molecular Catalysis, vol. 435, pp. 24-32, 2017.

[19] M. Sattari, A. Kamari, H. Hashemi, A. H. Mohammadi, and D. Ramjugernath, "A group contribution model for prediction of the viscosity with temperature dependency for fluorinecontaining ionic liquids," Journal of Fluorine Chemistry, vol. 186, pp. 19-27, 2016.

[20] J. J. Qi, J. Q. Lin, and H. Q. Fu, "One-step production of biodiesel from waste cooking oil catalysed by $\mathrm{SO}_{3} \mathrm{H}$-functionalized quaternary ammonium ionic liquid," Current Science, vol. 110, no. 11, pp. 2129-2134, 2016.

[21] R. A. Molla, P. Bhanja, K. Ghosh, S. S. Islam, A. Bhaumik, and S. M. Islam, "Pd nanoparticles decorated on hypercrosslinked microporous polymer: a highly efficient catalyst for the formylation of amines through carbon dioxide fixation," ChemCatChem, vol. 9, no. 11, pp. 1939-1946, 2017.

[22] X. Li, C. Lv, X. Jia, M. Cheng, K. Wang, and Z. Hu, "Nanoparticle based on poly(ionic liquid) as an efficient solid immobilization catalyst for aldol reaction and multicomponent reaction in water," ACS Applied Materials \& Interfaces, vol. 9, no. 1, pp. 827-835, 2016.

[23] J. J. Boruah, K. Ahmed, S. Das et al., "Peroxomolybdate supported on water soluble polymers as efficient catalysts for green and selective sulfoxidation in aqueous medium," Journal of Molecular Catalysis A Chemical, vol. 425, pp. 21-30, 2016.

[24] M. Yoshizawa, M. Hirao, K. Ito-Akita, and H. Ohno, "Ion conduction in zwitterionic-type molten salts and their polymers," Journal of Materials Chemistry, vol. 11, no. 4, pp. 1057-1062, 2001.
[25] A. L. Robinson, H. J. Lee, and D. Ryu, "Polyvinylpolypyrrolidone reduces cross-reactions between antibodies and phenolic compounds in an enzyme-linked immunosorbent assay for the detection of ochratoxin A," Food Chemistry, vol. 214, pp. 47-52, 2017.

[26] V. Carrasco-Sánchez, G. Y. Kreitman, C. Folch-Cano, R. J. Elias, and V. F. Laurie, "Removal of fumonisin $B_{1}$ and $B_{2}$ from model solutions and red wine using polymeric substances," Food Chemistry, vol. 224, pp. 207-211, 2017.

[27] M. G. Chegini and M. Mokhtary, "Polyvinylpolypyrrolidonesupported chlorosulfonic acid: an efficient catalyst for one-pot synthesis of dihydropyrimidinones and octahydroquinazolin-2,5-diones," Polycyclic Aromatic Compounds, vol. 37, no. 1, pp. 63-72, 2016.

[28] S. Wang, Q.-Q. Chang, N. Shawgi, S.-X. Li, and L.-N. Zhang, "Synthesis of butyrate using a heterogeneous catalyst based on polyvinylpolypyrrolidone," Chemical Papers, vol. 70, pp. 538-544, 2016.

[29] S. Wang and C. Tian, "Polyvinylpolypyrrolidone supported Brønsted acidic catalyst for esterification," International Journal of Polymer Science, vol. 2016, Article ID 8104838, 7 pages, 2016.

[30] H. C. Nguyen, S. H. Liang, T. T. Doan, C. H. Su, and P. C. Yang, "Lipase-catalyzed synthesis of biodiesel from black soldier fly (Hermetica illucens): optimization by using response surface methodology," Energy Conversion \& Management, vol. 145, pp. 335-342, 2017.

[31] S. A. Pasma, R. Daik, M. Y. Maskat, and O. Hassan, "Application of Box-Behnken design in optimization of glucose production from oil palm empty fruit bunch cellulose," International Journal of Polymer Science, vol. 2013, Article ID 104502, 8 pages, 2013.

[32] S. Matiacevich, N. Riquelme, and M. L. Herrera, "Conditions to prolonged release of microencapsulated carvacrol on alginate films as affected by emulsifier type and $\mathrm{PH}$," International Journal of Polymer Science, vol. 2015, Article ID 173193, 12 pages, 2015.

[33] Y. Leng, P. Jiang, and J. Wang, "A novel Brønsted acidic heteropolyanion-based polymeric hybrid catalyst for esterification," Catalysis Communications, vol. 25, pp. 41-44, 2012.

[34] S. Wang, L. Xu, P. U. Okoye, S. Li, and C. Tian, "Microwaveassisted transesterification of glycerol with dimethyl carbonate over sodium silicate catalyst in the sealed reaction system," Energy Conversion and Management, vol. 164, pp. 543-551, 2018.

[35] P. U. Okoye, A. Z. Abdullah, and B. H. Hameed, "Stabilized ladle furnace steel slag for glycerol carbonate synthesis via glycerol transesterification reaction with dimethyl carbonate," Energy Conversion and Management, vol. 133, pp. 477-485, 2017.

[36] M. S. Gruzdev, L. M. Ramenskaya, U. V. Chervonova, and R. S. Kumeev, "Preparation of 1-butyl-3-methylimidazolium salts and study of their phase behavior and intramolecular intractions," Russian Journal of General Chemistry, vol. 79, no. 8, pp. 1720-1727, 2009.

[37] H. Luo, H. Yin, R. Wang, W. Fan, and G. Nan, "Caprolactambased Brønsted acidic ionic liquids for biodiesel production from jatropha oil," Catalysts, vol. 7, no. 12, 2017.

[38] M. Vafaeezadeh and M. M. Hashemi, "Efficient fatty acid esterification using silica supported Brønsted acidic ionic liquid catalyst: experimental study and DFT modeling," Chemical Engineering Journal, vol. 250, pp. 35-41, 2014. 


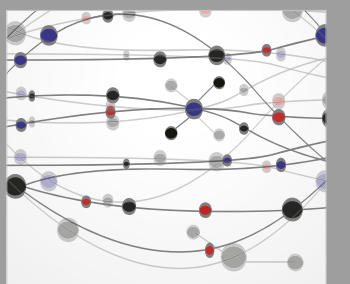

The Scientific World Journal
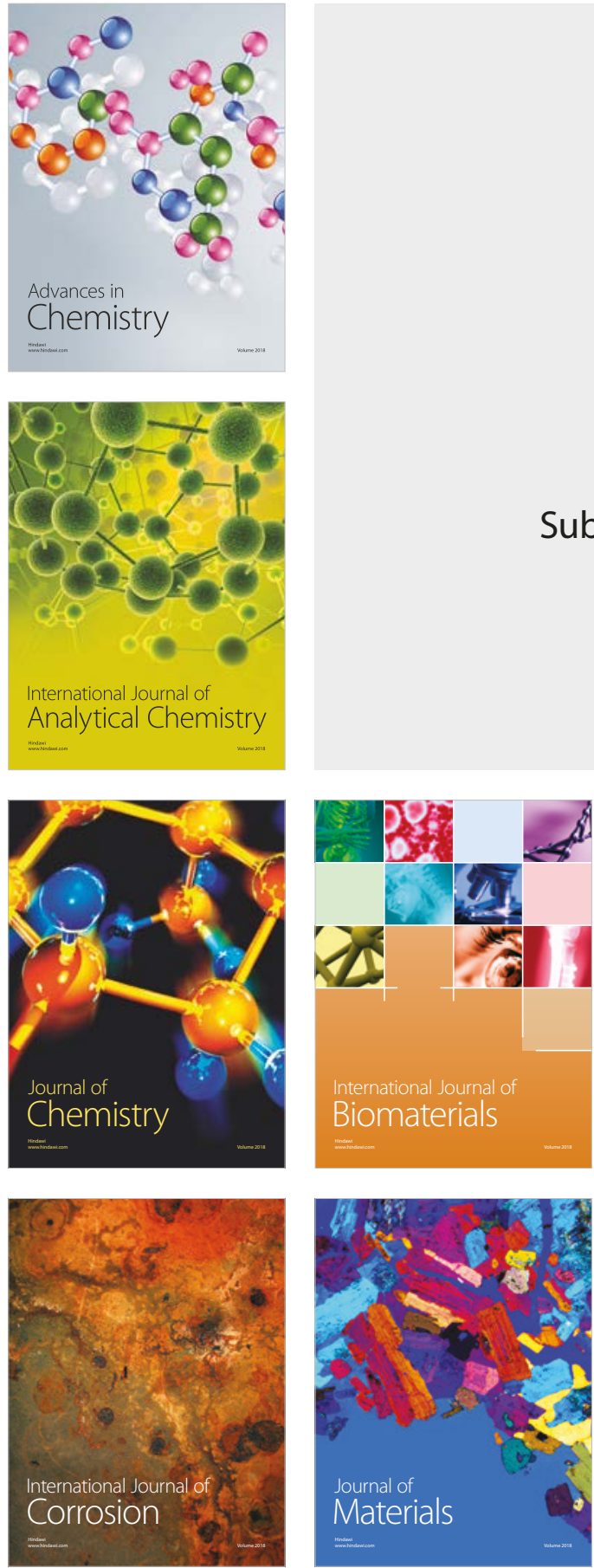

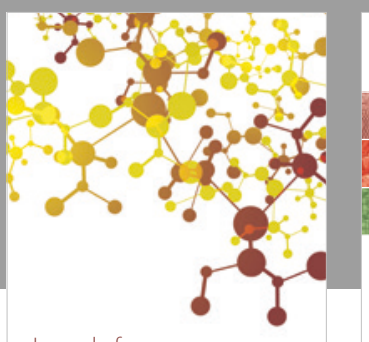

Journal of

Applied Chemistry
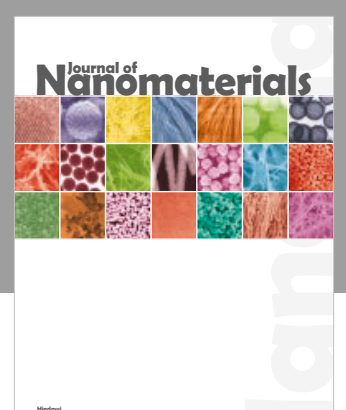

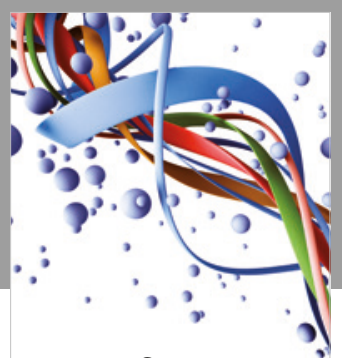

Scientifica

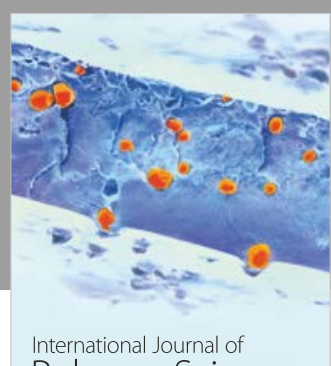

Polymer Science

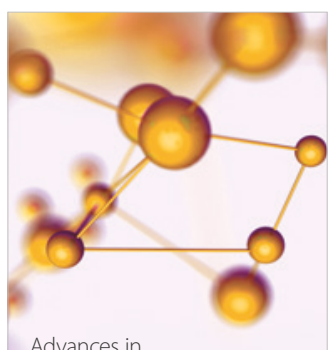

Physical Chemistry
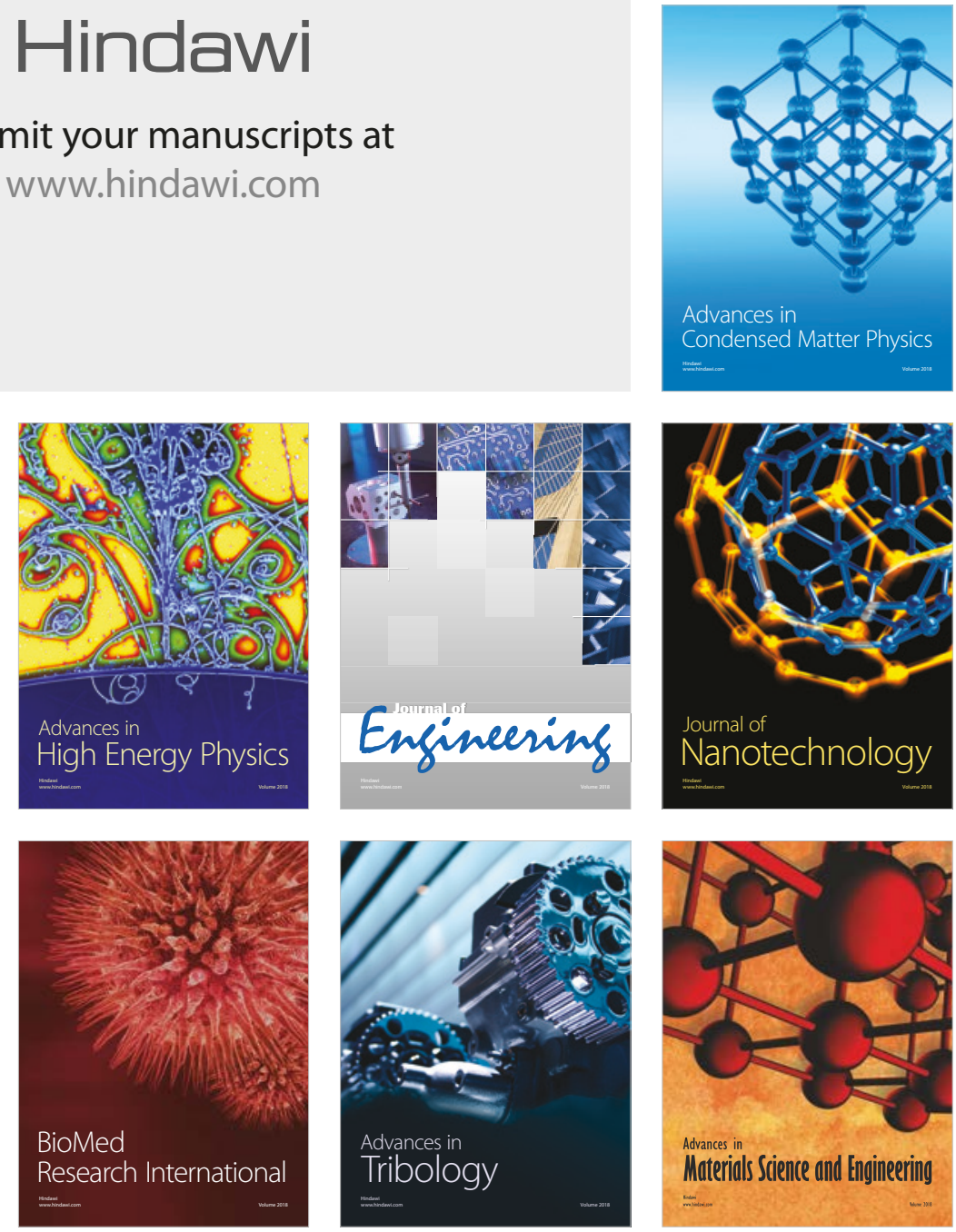